\title{
Sensor solutions for an energy-efficient and user-centered heating system
}

\author{
Moritz Hein ${ }^{1}$, Ralf Stöber ${ }^{1}$, Michael Meiler ${ }^{1}$, Daniel Schaller ${ }^{1}$, Rebecca Zehle ${ }^{1}$, Gerhard Fischerauer ${ }^{1}$, \\ Jochen Bauer $^{2}$, Johannes Bürner ${ }^{2}$, Jörg Franke ${ }^{2}$, Thomas Becher ${ }^{3}$, Martin Feller $^{4}$, and Joachim Maul ${ }^{5}$ \\ ${ }^{1}$ Chair of Measurement and Control Systems and Center of Energy Technology (ZET), University of Bayreuth, \\ 95440 Bayreuth, Germany \\ ${ }^{2}$ Institute for Factory Automation and Production Systems, Friedrich-Alexander-Universität \\ Erlangen-Nürnberg, 91058 Erlangen, Germany \\ ${ }^{3}$ TBE-Hof, 95028 Hof, Germany \\ ${ }^{4}$ Frenzelit Werke GmbH, 95460 Bad Berneck, Germany \\ 5 ait-deutschland GmbH, 95359 Kasendorf, Germany \\ Correspondence to: Moritz Hein (mrt@uni-bayreuth.de)
}

Received: 30 September 2016 - Accepted: 19 December 2016 - Published: 23 January 2017

\begin{abstract}
In contrast to conventional hydronic heating systems, in which the air is used as a medium for the convective heat transfer, an alternative approach is based on the usage of infrared (IR) radiant heating foils. These foils, which are applied to the walls and the ceiling of a laboratory, can be controlled individually. This leads to the possibility of heating the room zonewise and only when a person is present in a zone. A local comfortable climate is provided only in occupied zones, with the remaining zones being kept at a lower base temperature. Consequently, the measurement system has to detect persons in each zone and to determine the putative thermal comfort at relevant locations in the room. For the first problem, we examined and evaluated different sensor types capable of localizing persons without infringing on their anonymity. For the second problem, we used the fact that the thermal comfort mainly depends on the operative temperature (Li et al., 2010; DIN EN ISO 7730, 2006; de Dear and Brager, 2002). According to Simone et al. (2007), this temperature can be measured directly by an easily producible, planar sensor. The sensors were integrated in a wireless sensor network which consists of Wi-Fi-capable microcontroller boards, wireless smart home equipment, a Wi-Fi router, and a server.
\end{abstract}

\section{Introduction}

The challenge when designing a heating system is that it should be as energy efficient as possible and yet must provide a comfortable room climate for the occupants and take their wishes and needs concerning the usage of the system into consideration (Hein et al., 2016a). These requirements have been addressed by Braun et al. (2016) by applying copper traces in the shape of a meander to the walls and supplying them with electrical energy. The system was operated in such a way that the surface temperature was about $28^{\circ} \mathrm{C}$, which was seemingly sufficient to provide a comfortable room climate. There have been studies concerning the combination of heat pumps with photovoltaic systems (Brunner et al., 2016) and even field trials with retrofitted heat pumps operated with high water temperatures (Shah and Hewitt, 2015). Crucial to such systems is the overall management of the various components, which needs to be intelligent at the data acquisition and control level (Chen et al., 2015; Fernandes et al., 2015).

By theoretical reasoning and by experiments, we addressed the following issues:

- Which functional units are required to build a smart room climate control, and what is a suitable system architecture?

- The system will need to comprise several sensors (temperature, localization, etc.). This places financial restrictions on the realization. 


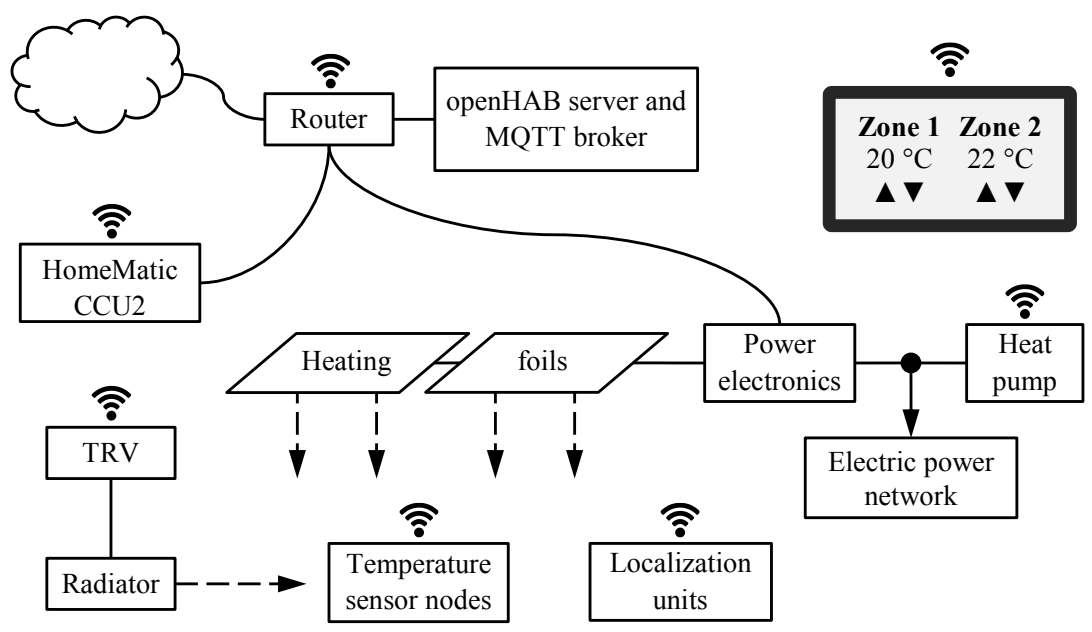

Figure 1. Heterogeneous architecture of the user-centered heating system. The communication is built around a Wi-Fi-capable router which is connected to the internet. The microcontrollers, which are used as temperature sensor nodes, as well as the controllers for the power electronics communicate via the resource-friendly message queue telemetry transport (MQTT) protocol. The HomeMatic components, like the thermostatic radiator valves (TRV), are accessible with the HomeMatic CCU2 Gateway. Users can interact through tablets, for instance; their commands, together with the sensor and actuator data, are stored and processed on the server and furthermore distributed in the network.

- What are the relevant deviations between the characteristics of the functional units and the physical units used to implement the functional-unit characteristics?

- How can the technical communication between the various physical units of the heating system (sensors, heating elements, controllers) be organized? The resulting system can be expected to be very heterogeneous, including different data formats and communication standards.

\section{Architecture}

Because of the individual requirements of the inhabitants and the differing constraints of the buildings where the heating system will be built in, the overlaying management system should be as open and flexible as possible. In the past, smart home system providers offered vendor-dependent solutions and are recently trying to create vendor-specific alliances. In contrast to that, users prefer vendor independence because they want to add devices of all available providers, if those are relevant for their use cases. Therefore, a middleware system or an accepted standard is necessary. Currently, there are promising concepts for user-friendly middleware platforms, like openHAB, Eclipse-SmartHome, or Qivicon offered by Telekom, which share the same conceptual ideas regarding their technology stack. The framework used by us for the operation of the measuring and control system is openHAB 2, an open-source framework which has gained more and more attention in the scientific community (Smirek et al., 2016). As usual, for middleware platforms like openHAB, a user interface, device abstraction, and opportunities to use different

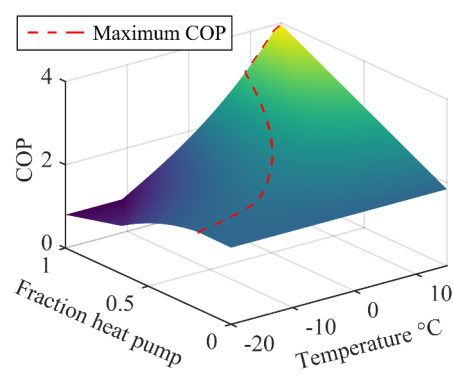

Figure 2. Simulation of the coefficient of performance (COP) of the combined heating system with the COP over the fraction of energy provided by the heat pump (the remaining necessary energy is provided by the heating foils) and the outdoor temperature.

communication protocols are available. The openHAB system follows the OSGi specification, and therefore it is possible to create plugins, called add-ons, which extend current functionality and can be enabled or disabled at runtime. One common use case for such an add-on is to add a translation layer for another communication protocol. In the openHAB ecosystem, those translators are called bindings. There are more than 100 bindings available (in openHAB), so lots of technologies can be used, i.e., online weather services, smart light bulbs, and a variety of communication protocols like KNX, Z-Wave, and Modbus.

Based on that framework, we had to divide the overall problem into smaller functional units, which are the heating system, the indoor localization of inhabitants, the thermal comfort measurement, and the user interface. Their resemblance as physical units was derived and finally combined to the overall system (Fig. 1). 


\subsection{Heating system}

The redevelopment of old building stock is feasible in different ways. Obvious and widespread building measures are the exchange of the old windows by better insulated ones or the application of an additional layer of building insulation (BDEW, 2015). But there are buildings where such measures are not applicable, e. g., listed buildings. Therefore, we have examined the combination of two heating technologies, which can be retrofitted in existing buildings, and the resulting synergy. In this concept, the existing hydronic system is detached from the old source of heat (gas or oil burner) and equipped with a heat pump. To take advantage of the heat pump's high coefficient of performance (COP), the amount of thermal energy provided by the heat pump is limited depending on the outdoor air temperature. The remaining share of energy is provided by infrared (IR)-radiant heating foils, which operate relatively independent of the weather conditions and are therefore on a constant level of performance. The simulation of this combined system, by varying the outdoor temperature and the fraction of energy provided by each heat source, shows that there exists an optimum of operation at every temperature on the interval (Fig. 2). If the weather is mild, the heat pump drives the heating system exclusively. The colder it gets, the more energy is provided by the heating foils. With such a simulation, the performance of the heating system is predictable for varying annual average temperatures in different regions.

The heating foils, which are powered by switching power supplies, are placed at the ceiling evenly and establish a comfortable room climate where necessary. The fact that the heating foils can be switched individually enables one to create different temperature zones in a room. But this also requires solutions for a zone-wise measurement of the occupants' thermal comfort and their location in the room. This measurement problem (selection of measurands, characterization of individual sensors, heterogeneous integration into a control system) is the subject of this paper (Hein et al., 2016b) and the following sections.

\subsection{Indoor localization}

The localization of an occupant is crucial for a lot of smart home use cases. There are several strategies to obtain this kind of information, i.e., Bluetooth, RFID, infrared, or ultrasonic sensors (Deak et al., 2012). In the context of usercentered heating scenarios, the following questions are relevant:

- Is it necessary to identify an occupant?

- Are camera-based solutions acceptable to occupants?

- Does a given solution measure precisely and accurately enough?

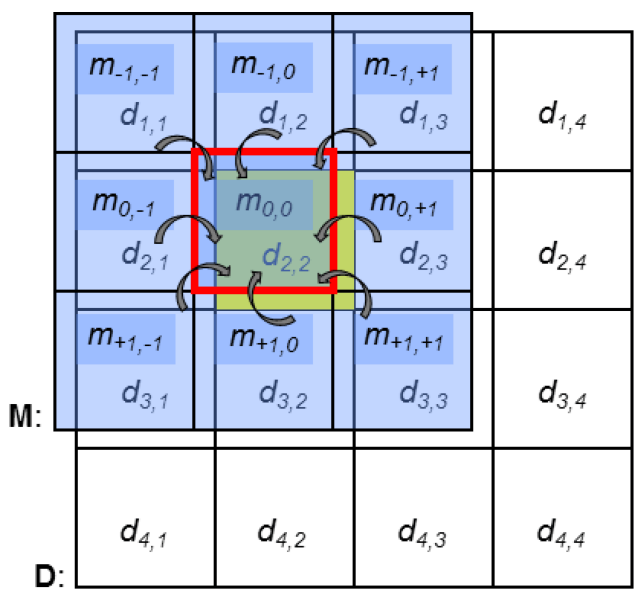

Figure 3. Signal processing for the thermopile array by dynamic masking (mask M) of the data points (matrix D). The maximum heat radiation was detected by pixel $d_{2,2}$ (yellow).

Table 1. Excerpt of the output data of an experiment with six persons; row and column of sensor pixels and the resulting presence (1) and absence $(0)$ of persons in the field of view of the listed pixels.

\begin{tabular}{llll}
\hline Row & Column & Value $\left({ }^{\circ} \mathrm{C}\right)$ & Presence \\
\hline 1 & 2 & 19.4 & 0 \\
2 & 1 & 23.9 & 0 \\
3 & 2 & 29.3 & 1 \\
4 & 1 & 24.0 & 0 \\
\hline
\end{tabular}

- Would an occupant agree to wearing some kind of device?

- Are the installation costs economically justifiable?

- Is the installation possible in existing houses?

There exists a variety of sensors for the indoor localization of persons in a heating zone. Owing to privacy and acceptance concerns (Arning and Ziefle, 2015), image generating and processing systems, like cameras, have been ruled out. Radiating sensors, like radar or ultrasonic sensors, would be suitable, but it is highly questionable in many societies, and certainly in Germany, whether users would accept them in the living room or in the bedroom.

For this reason, we focused our research on passive sensors like pyroelectric infrared sensors (PIRs), thermopiles, thermopile arrays, and $\mathrm{CO}_{2}$ sensors. Their suitability for the anonymous localization differs, and thermopiles turned out to yield the overall best results for different situations. The PIR sensors only respond to heat flux changes and thus can detect moving persons but not persons at rest. $\mathrm{CO}_{2}$ sensors react to an increase of $\mathrm{CO}_{2}$ in the air which is attributable to the presence of persons in a room. Unfortunately, the time delay for even small rooms is quite big. Consequently, these 

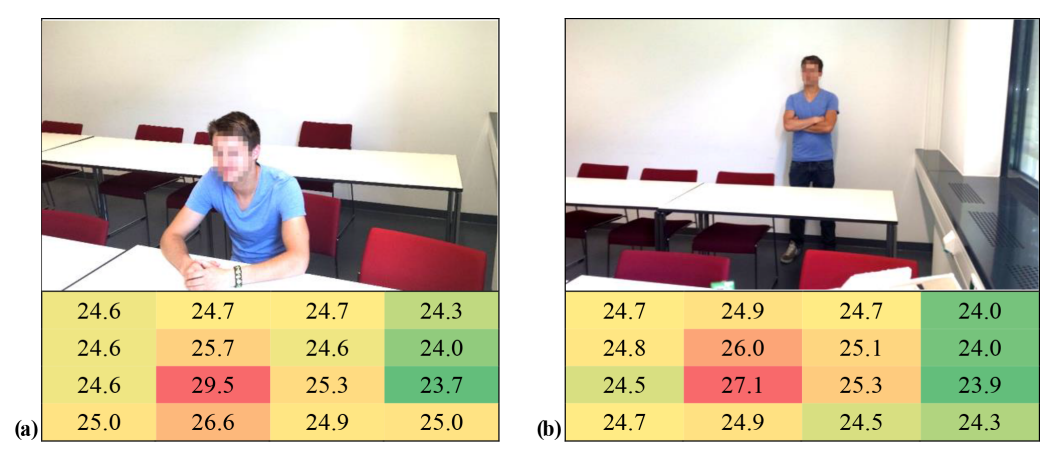

Figure 4. Output of the $4 \times 4$ thermopile array with (a) a sitting person and (b) a standing person. Measured temperatures are in ${ }^{\circ} \mathrm{C}$. The photos shown convey an idea of the scenarios the thermopile array was subjected to.
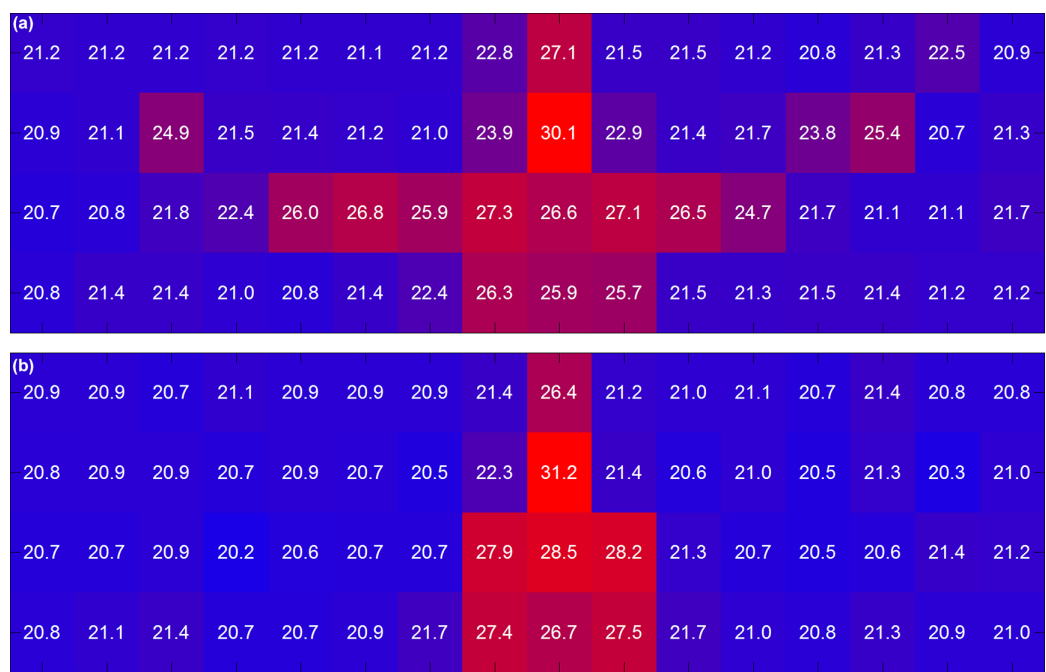

Figure 5. Experimental results with a $16 \times 4$ thermopile array pointed towards a person with (a) arms spread out and (b) arms at the sides of the body. Values are in ${ }^{\circ} \mathrm{C}$.

sensors were not investigated in more detail. For the experiments reported in the following, we used the thermopile array Omron D6T-44L-06 as an IR-radiation-based means of signaling the presence of persons in a heating zone.

A possible spatial distribution of the sensors for a maximum field of view and a reliable detection has already been described in Kuki et al. (2013). Four sensors were arranged in a square configuration to obtain an $8 \times 8$ pixel resolution. On the assumption that a person fills up $3 \times 3$ pixels at most, the position was determined. So, for a precise localization within a room, several arrays are necessary. However, when one is mainly interested in the presence detection per heating zone, as we are, a single sensor suffices.

By analogy with image processing methods, the sensor output data are arranged in a $4 \times 4$ matrix $\mathbf{D}$ which resembles the sensor (thermopile array) structure. After that, the pixels with minimum and maximum received IR radiation are identified as $d_{\max }=\max \left(d_{i, j}\right)$ and $d_{\min }=\min \left(d_{i, j}\right)$ with pixel temperatures $T_{\max }$ and respectively $T_{\min }$. The algorithm which decides whether a person is present or not is based on the following premises.

The determined maximum $T_{\max }$ must not be greater than $34^{\circ} \mathrm{C}$. No part of the human body has a higher surface temperature.

The determined minimum $T_{\min }$ has to be close to the enveloping surface temperature; otherwise, no distinction between the person and other heat-radiating objects can be made in the room.

To suppress random noise, $T_{\max }-T_{\min } \geq 2{ }^{\circ} \mathrm{C}$ must apply.

Finally, a $3 \times 3$ mask $\mathbf{M}$ is placed on the coordinates of $d_{\max }$ and the surrounding eight pixels, inheriting the temperature values of $\mathbf{D}$ with $T_{\max }$ at $m_{0,0} . T_{\max }$ is then compared to the surrounding values (Fig. 3). When the deviation is big enough, but not too big, the probability that a person is present rises. If the values of two or more pixels are on this temperature interval, a person is regarded as detected.

At first, several experiments with a person at varying distances from the sensor were carried out to validate the algorithm. The person was detected reliably despite the high out- 
door temperature of $30^{\circ} \mathrm{C}$ (Fig. 4). The sensor and algorithm worked well even with a group of six people sitting in front of the sensor with a distance of $2 \mathrm{~m}$, which was confirmed by a positive presence feedback in row 3 of Table 1 .

Among the possible influencing effects are pets or other IR-radiating objects, such as a kettle. They could lead to a false positive presence detection and finally to the activation of the control loop. The sensitivity of the algorithm to such effects was characterized by experiments. It was found that the difference of the temperature of animal fur and the surface temperature of clothing is quite small. Only the temperature of the human skin of approximately $34^{\circ} \mathrm{C}$ makes a significant difference to the surface fur temperature of $26^{\circ} \mathrm{C}$. Animals smaller than cats were not mistakenly detected as human beings. Cats and dogs with shorter fur, and therefore a higher surface temperature, on the other hand, were detected. Another situation in which the algorithm erroneously signaled the presence of a person was when animals were moving to or from the sensor. A dog with a height of $60 \mathrm{~cm}$ and very long fur, hence low surface temperature, caused false positives only rarely. Other disturbances like kettles, monitors, or even windows, which were warmed up by the insolation, did not lead to wrong alarms. Nonetheless, we observed that the algorithm only very rarely detected a (4-year old) child as a person.

In an attempt to increase the resolution, we examined the thermopile array Melexis MLX90621, which comprises $16 \times 4$ pixels. In contrast to the Omron D6T-44L-06, the higher resolution leads to the possibility to recognize a person's shape (Fig. 5) and thus reduce the probability of false positives. Another advantage of the higher resolution is that the number of persons in a room can be determined. In comparison to cameras, the resolution is low enough to maintain the occupants' anonymity. Although the advantages of the thermopile arrays over PIR sensors are obvious, their higher purchase price is a major drawback in view of the fact that at least one sensor per room is needed. Our studies with commercial motion sensors (which use a PIR sensor internally) in an office show that the presence of persons who are working at their desks, even if practically at rest, is detectable.

\subsection{Measurement of thermal comfort}

The goal of a user-centered heating system is the occupants' subjective sensation of heat or, in other words, their sense of thermal comfort. Consequently, this sensation has to be estimated by a technically measurable quantity (Aswani et al., 2012). To this end, the predicted mean vote (PMV) has been established in the ISO standard 7730 (DIN EN ISO 7730, 2006). This standard has several drawbacks, e.g., the complex and only iteratively solvable calculation of the PMV and the dependency on poorly measurable quantities, such as the clothing level and the metabolic rate of a person. We therefore considered the operative temperature as a possible controlled variable. The comfortable operative temperature

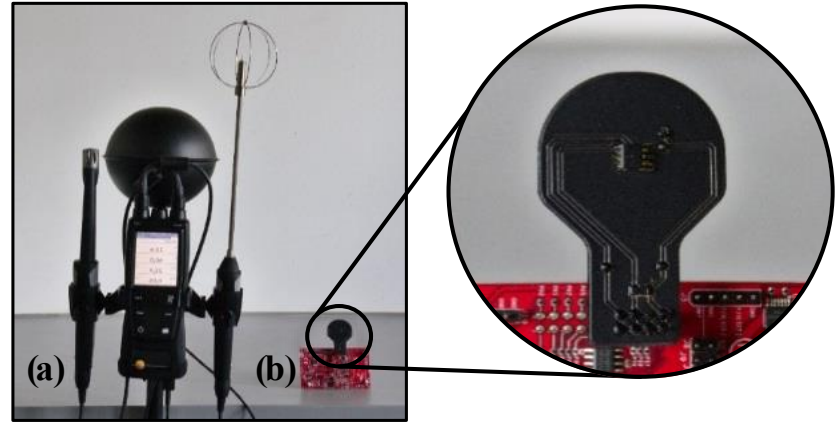

Figure 6. Sensors investigated: (a) digital temperature and humidity meter Testo 480 with probes to measure the air temperature and humidity, the globe temperature (black copper sphere with a settling time of $20 \mathrm{~min}$ ), and the air velocity; (b) sensor attached to a microcontroller board.

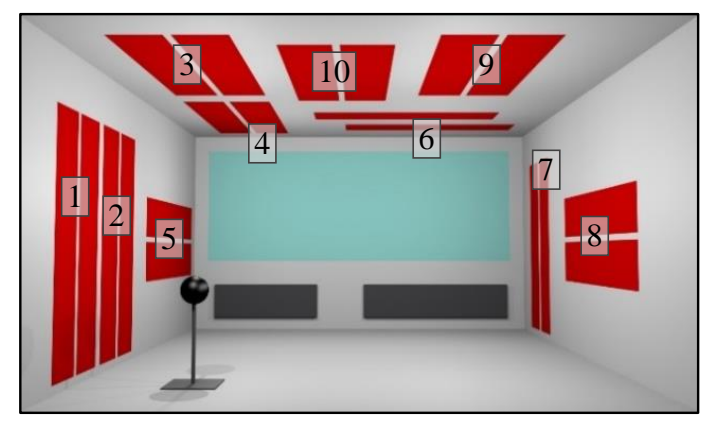

Figure 7. Arrangement of the heating foils in the demonstrator room (red). The black sphere marks the position of the globe thermometer or the single temperature sensor.

$T_{\text {comf }}$ is determined on the basis of the outdoor air temperature (de Dear and Brager, 2002; Nicol and Humphreys, 2010) which should satisfy most of the occupants. $T_{\text {comf }}$ is calculated as follows (Nicol and Humphreys, 2010):

$T_{\mathrm{comf}}=0.331 \cdot T_{\mathrm{rm}}$,

where $T_{\mathrm{rm}}$ is the running mean temperature

$T_{\mathrm{rm}}=0.2 \cdot T_{\mathrm{dm}-1}+0.8 \cdot T_{\mathrm{rm}-1}$.

Here, $T_{\mathrm{dm}-1}$ and $T_{\mathrm{rm}-1}$ are the previous-day values for the $24 \mathrm{~h}$ daily mean outdoor temperature and the running mean outdoor temperature. For outdoor temperatures below $10^{\circ} \mathrm{C}$, $T_{\text {comf }}$ is considered constant with a value of $22^{\circ} \mathrm{C}$.

The operative temperature can be determined by a climatemeasuring instrument - we used the type Testo 480 - which comprises a probe for the air velocity, a globe thermometer, and an air thermometer. At a typical indoor air velocity of $v \leq 0.1 \mathrm{~m} \mathrm{~s}^{-1}$, the operative temperature $T_{\mathrm{op}}$ is the arithmetic mean of the indoor air temperature $T_{\mathrm{a}}$ and the mean radiant temperature $T_{\mathrm{r}}$ :

$T_{\mathrm{op}}=\frac{1}{2}\left(T_{\mathrm{a}}+T_{\mathrm{r}}\right)$. 


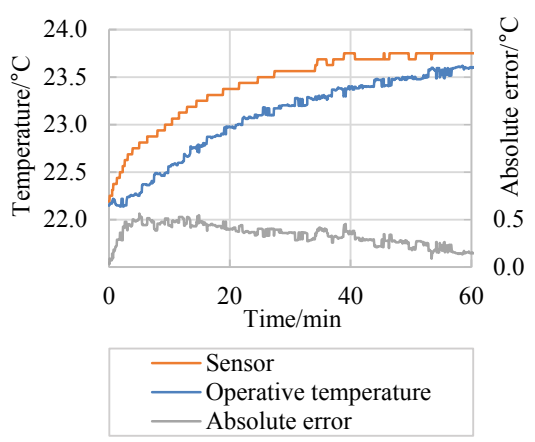

Figure 8. Step response of the various temperatures at a selected location in the demonstrator room after turning heating foil pair 1 on.

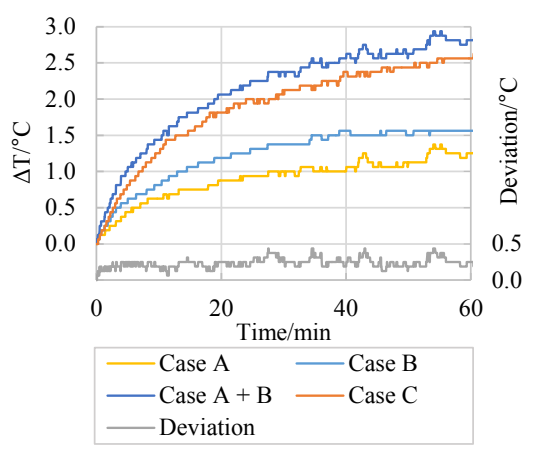

Figure 9. Comparison of the measured temperature changes in case A (pair 3 powered), in case B (pair 1 powered), and in case C (pairs 1 and 3 powered simultaneously). Also shown is the calculated sum of the results of cases A and B ("Case A+B") and the deviation of this calculation from the measurement in case $C$.

$T_{\mathrm{r}}$ is calculated in the following manner according to ISO 7726 (ISO 7726, 1998):

$$
\begin{aligned}
& T_{\mathrm{r}} /{ }^{\circ} \mathrm{C}=\left[\left(T_{\mathrm{g}} /{ }^{\circ} \mathrm{C}+273\right)^{4}+k \cdot\left(T_{\mathrm{g}}-T_{\mathrm{a}}\right) /{ }^{\circ} \mathrm{C}\right]^{0.25}-273, \\
& k=\frac{1.1 \cdot 10^{8} \cdot\left(v /\left(\mathrm{m} \mathrm{s}^{-1}\right)\right)^{0.6}}{\varepsilon_{\mathrm{g}} \cdot\left(d_{\mathrm{g}} / \mathrm{m}\right)^{0.4}},
\end{aligned}
$$

with the globe, indoor air, and mean radiant temperatures $T_{\mathrm{g}}$, $T_{\mathrm{a}}$, and $T_{\mathrm{r}}$, respectively. $v$ is the air velocity, and $\varepsilon_{\mathrm{g}}=0.95$ and $d_{\mathrm{g}}=0.15 \mathrm{~m}$ are the emission coefficient and the diameter of the globe thermometer, respectively.

As the climate-measuring instrument cannot be deployed in living spaces for size and cost reasons, we examined a round, planar temperature sensor with a diameter of $30 \mathrm{~mm}$ (Fig. 6) as suggested by Simone et al. (2007) as an alternative. Experiments in a room of approximately $40 \mathrm{~m}^{2}$ equipped with 10 pairs of heating foils (Fig. 7) showed that, at steady-state operation (constant temperature), such a sensor provides nearly the operative temperature calculated from the output values of the climate-measuring instrument. In dynamic experiments, in which heating foils were turned on

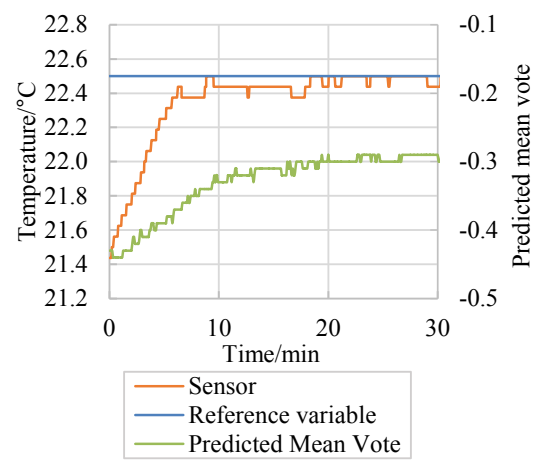

Figure 10. Step responses of the operative temperature and of the PMV at a selected location in the demonstrator room in a closedloop experiment with heating foil pairs 3 and 10 used as actuators.

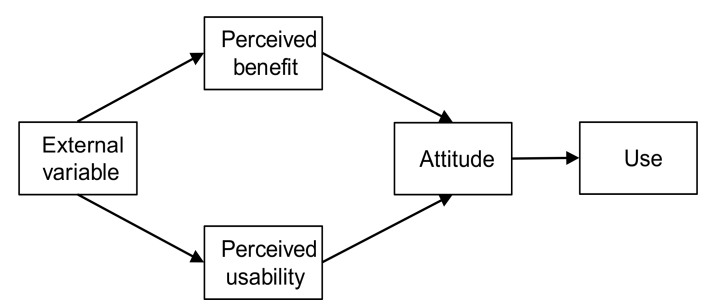

Figure 11. Technology acceptance model (Davis et al., 1986).

and the resulting transient heating processes were monitored, the sensor reacted faster than the climate-measuring instrument, owing to its smaller thermal mass and therefore shorter settling time (Fig. 8). Another result of the dynamic experiments, also apparent in Fig. 8, is that the IR heating system has a time constant of 10 to $20 \mathrm{~min}$. It is clear that such a sluggish behavior has to be taken into account in the design of the temperature feedback control.

This design is also influenced by the linear or nonlinear nature of the controlled system. We carried out experiments to determine if the combined action of several heating foils can be described by the sum of the actions of the individual foils when operated alone (in other words, if the system can be treated as linear; the system is nonlinear, as shown by Eq. (4), but small temperature changes should warrant a linearization). This is indeed the case. For instance, the difference between the added individual actions of two pairs of heating foils and their combined simultaneous action was less than $0.5^{\circ} \mathrm{C}$ (Fig. 9). It follows that the whole system can be characterized by measuring the individual actions of the heating foils.

We used a digital PI controller to control the operative temperature and examined the response to a step in the reference variable from 21.5 to $22.5^{\circ} \mathrm{C}$ (Fig. 10). After approximately $5 \mathrm{~min}$, the control difference was only $0.1^{\circ} \mathrm{C}$, without any overshoot. In the time response of the PMV, the long settling time of the globe thermometer (approximately $20 \mathrm{~min}$ ) became apparent again. Its usage as measuring element would 


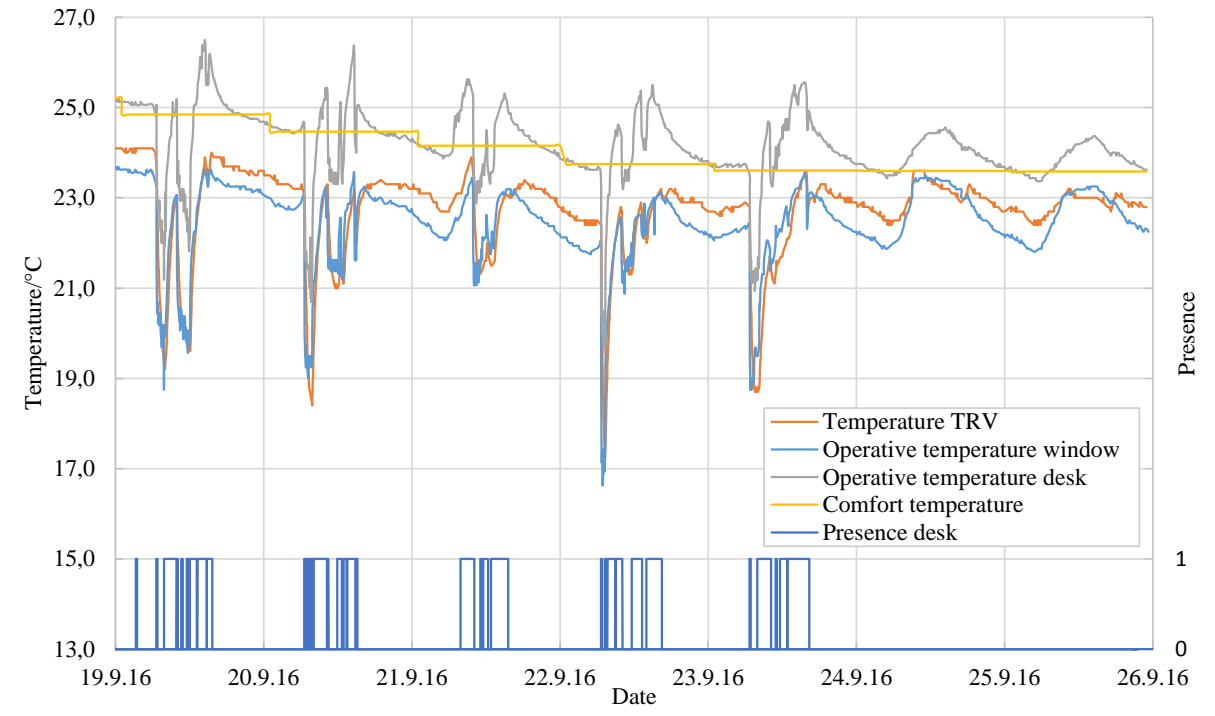

Figure 12. Time series of measured temperatures and occupant presence in a naturally ventilated office over a period of 7 days. The comfortable operative temperature was calculated from the measured values by Eq. (3).

increase the settling time of the feedback control system in comparison to the simple temperature sensor.

\subsection{User interface}

For the development of a new system, it is recommended to examine the acceptance or factors that increase the probability of use. In this context, acceptance is the positive reception of a chance a in physical environment after a deliberate examination of the change that has taken place (Renn, 1986). In the focus of the technology acceptance model, according to Davis (1986), are the perceived benefit and the perceived usability. As shown in Fig. 11, these two factors influence the attitude directly and thus also the use of an innovation.

In addition to the perceived benefit of the system, the perceived usability is the second crucial factor for the acceptance of a system. To ensure the usability, a humanmachine interface (HMI), which is adapted to the needs of its users, has to be designed and implemented. The user characteristics, as well as the technical and physical environment determine the context in which the system is applied (DIN EN ISO 9241, 2011). In former projects, our working group established a well-proven development process for user interfaces which consists of focus group interviews, card sorting, and usability testing (Bauer et al., 2014a). OpenHAB offers the possibility to design such an optimized user interface which is accessible by smartphones or web browsers. If the inhabitants are not familiar with such technologies, open$\mathrm{HAB}$ is flexible enough to embrace different solutions, like manual devices to set the desired temperature comparable to room thermostats.

\section{Architecture implementation and evaluation}

Our system has to comprise several temperature sensors, localization units, and possibilities for the inhabitants to interact with the system. Through openHAB bindings, different technologies, like MQTT sensor nodes equipped with our operative temperature sensor, HomeMatic components, and the control unit of the heat pump, are interconnected via a Wi-Ficapable router and managed by a small and energy-efficient PC. The PC is used as the central control unit (CCU) on which the openHAB server and a MQTT broker is running. The MQTT sensor nodes only send their measured values when the temperature change is bigger than $0.25^{\circ} \mathrm{C}$ or after 5 min without a connection to the broker. This approach is necessary to reduce the overall network traffic and to extend the lifetimes of the sensor batteries. In addition, we deployed various wireless smart home equipment such as HomeMatic thermostatic radiator valves (TRVs), motion detectors, sensors which detect opened and closed windows, room thermostats, and an outdoor temperature sensor. These wireless sensors and actuators communicate with the BidCoS standard via the HomeMatic CCU2. A subset of the incoming values are permanently stored in a time series database and thus are available for the calculation of the comfort temperature or other processing at a later point in time.

Bringing together the MQTT temperature sensor measurements and the ones by the HomeMatic components, Fig. 12 represents typical time series of temperatures and occupant presence in a naturally ventilated office (of approximately $20 \mathrm{~m}^{2}$ ). The employee started working around 09:00 CEST, when he also opened the window. The temperature decreased quickly until the window was closed. Even in such a small room, the difference in temperature of the sensor near the 
window and the one on the desk (distance $3 \mathrm{~m}$ ) is around $2{ }^{\circ} \mathrm{C}$. The longer settling time of the TRV is also evident in comparison to the operative temperature sensor nodes, especially when the room cooled down outside the working hours. In addition to the temperature progress, the measurements of the motion sensors were recorded. There is a clear distinction between the activity from 09:00 to 18:00 CEST and the rest of the day.

The digital controller is also implemented on the openHAB framework. Every $30 \mathrm{~s}$, the database is queried for the sensor and reference variable values, after which the controller output variables are calculated, and finally these variables are sent to the actuators. The reference variables are calculated from the values of wireless thermostats where the user can adjust the desired temperature in each room or, optionally, by a web interface and a mobile app. Furthermore, the energy supplying company can influence the room temperature within the bounds of the comfort temperature and with a lower priority than the user. This enables the supplying company to make use of load shifting and thus to use the available renewable energies more efficiently.

\section{Discussion}

We have analyzed the requirements of a smart heating system and, in particular, the necessary sensors and their integration in a feedback control system which relies on both wired and wireless communication. The system comprises temperature and localization sensors as measuring elements and heating foils as actuators. The latter were characterized experimentally, and the results of this characterization served to parameterize a feedback controller with the so-called operative temperature as the controlled variable because it is an accepted measure of thermal comfort. The system components were studied in a demonstrator room, and the entire heterogeneous system has now been deployed in an apartment for further field studies during the heating season of 2016/2017.

The localization of room occupants by thermopiles was shown to work reasonably well and is especially advantageous in terms of data security and user privacy. It does, however, allow no personalization of heating services. With those users who wish such a personalization in mind, it needs to be analyzed if personal identification could and should be added to the current implementation. It would then be possible to create individual profiles of thermal comfort for each occupant of a building. A way of implementing such functionality could be via an occupant's cell phone connected to the local home network.

Another possible extension of the demonstrator system could be to search the internet for relevant data to corroborate conclusions made by the system. An obvious choice would be to more consistently exploit weather forecasts. Such approaches, often implemented by using web services or endpoints, are promising strategies to benefit from the current trend to digitalization, often referred to as Internet of Things (Bauer et al., 2014b).

\section{Data availability}

The corresponding data set is available at doi:10.5281/zenodo.248977 (Hein et al., 2017).

Competing interests. The authors declare that they have no conflict of interest.

Acknowledgement. The authors would like to express their sincere thanks to the Bavarian State Ministry of Education, Science and the Arts for partially funding the presented work within the E|Home-Center.

Edited by: E. Starke

Reviewed by: two anonymous referees

\section{References}

Arning, K. and Ziefle, M.: "Get that Camera Out of My House!" Conjoint Measurement of Preferences for Video-Based Healthcare Monitoring Systems in Private and Public Places., 13th International Conference on Smart Homes and Health Telematics, Geneva, 10-12 June 2015, 152-164, doi:10.1007/978-3-31919312-0_13, 2015.

Aswani, A., Master, N., Taneja, J., Culler, D., and Tomlin, C.: Reducing Transient and Steady State Electricity Consumption in HVAC Using Learning-Based Model-Predictive Control, Proc. IEEE, 100, 240-253, doi:10.1109/JPROC.2011.2161242, 2012.

Bauer, J., Kettschau, A., and Franke, J.: Optimierung der Datenvisualisierung von AAL-Serviceplattformen durch Usability-Tests, 7. Deutscher AAL-Kongress, Berlin, 21-22 January 2014, 2014a.

Bauer, J., Kettschau, A., Michl, M., Bürner, J., and Franke, J.: Die intelligente Wohnung als Baustein im Internet der Dinge: Potenzialanalyse und Konzept einer domänenübergreifenden Lösung, Conference Technische Unterstützungssysteme, die die Menschen wirklich wollen, Hamburg, 15-16 December 2014, 12 pp., doi:10.13140/2.1.4173.0886, 2014b.

BDEW Bundesverband der Energie- und Wasserwirtschaft e. V.: Wie heizt Deutschland? - BDEW-Studie zum Heizungsmarkt, BDEW Bundesverband der Energie- und Wasserwirtschaft e. V., p. 28, 2015.

Braun, T., Bürner, J., Michl, M., Schaller, L., Böhm, R., and Franke, J.: Innovative Flexible Heating System by the Use of Additive Plasma Coating Technology- Heating Where Heat Is Needed by Additive Metallization of Furniture and Walls, 4th IEEE International Conference on Smart Energy Grid Engineering, Oshawa, 21-24 August 2016, 278-283, 2016.

Brunner, M., Rudion, K., and Tenbohlen, S.: PV curtailment reduction with smart homes and heat pumps, IEEE International Energy Conference, Leuven, 4-8 April 2016, 6 pp., doi:10.1109/ENERGYCON.2016.7514075, 2016. 
Chen, X., Yang, S.-H., Wong, C. B., Moore, P., and Chen, J.: Domestic space heating energy control via Smart Home Energy Management. IEEE International Conference on Mechatronics and Automation, Beijing, 2-5 August 2015, 905-911, doi:10.1109/ICMA.2015.7237606, 2015.

Davis, F. D.: A Technology Acceptance Model for Empirically Testing New End-User Information Systems: Theory and Results, Cambridge, MIT Sloan School of Management, Massachusetts Institute of Technology, 24-26, 1986.

Deak, G., Curran, K., and Condell, J.: A survey of active and passive indoor localisation systems, Comput. Commun., 35, 1939-1954, doi:10.1016/j.comcom.2012.06.004, 2012.

de Dear, R. and Brager, G.: Thermal comfort in naturally ventilated buildings: revisions to ASHRAE Standard 55, Energ. Buildings, 34, 549-561, doi:10.1016/S0378-7788(02)00005-1, 2002.

DIN EN ISO 7730:2006-05, Ergonomie der thermischen Umgebung - Analytische Bestimmung und Interpretation der thermischen Behaglichkeit durch Berechnung des PMV- und des PPDIndexes und Kriterien der lokalen thermischen Behaglichkeit, DIN Deutsches Institut für Normung e.V., p. 6, 2006.

DIN EN ISO 9241-210:2011-01, Ergonomie der Mensch-SystemInteraktion - Teil 210: Prozess zur Gestaltung gebrauchstauglicher, interaktiver Systeme, DIN Deutsches Institut für Normung e.V., p. 14, 2011.

Fernandes, Fi., Carreiro, A., Morais, H., Vale, Z., Gastaldello, D. S., do Amaral, H. L. M., and Nunes de Souza, A.: Management of Heating, Ventilation and Air Conditioning system for SHIM platform. IEEE PES Innovative Smart Grid Technologies Latin America, Montevideo, 5-7 October 2015, 275-280, doi:10.1109/ISGT-LA.2015.7381167, 2015.

Hein, M., Stöber, R., Fischerauer, G., Bauer, J., Bürner, J., Kettschau, A., Franke, J., and Feller, M.: Heating system to provide a comfortable indoor climate and to reduce negative effects on health in old buildings, Zukunft Lebensräume Kongress, Frankfurt a. M., 20-21 April 2016, 102-108, 2016a.

Hein, M., Stöber, R., Zehle, R., Meiler, M., Schaller, D., Fischerauer, G., and Feller, M.: Sensorlösungen für eine energieeffiziente nutzerzentrierte Heizung, Tagungsbd. 18. GMA/ITGFachtagung Sensoren und Messsysteme, Nürnberg, 10-11 May 2016, 663-668, doi:10.5162/sensoren2016/P5.7, 2016 b.
Hein, M., Meiler, M., Schaller, D., and Zehle, R.: Sensor solutions for an energy-efficient and user-centered heating system, doi:10.5281/zenodo.248977, 2017.

ISO 7726:1998, Ergonomics of the thermal environment - instruments for measuring physical quantities, International Organization for Standardization, Geneva, Switzerland, p. 19, 1998.

Kuki, M., Nakajima, H., Tsuchiya, N., Kuramoto, K., Kobashi, S., and Hata, Y.: Mining Multi Human Locations Using Thermopile Array Sensors, IEEE 43rd International Symposium on MultipleValued Logic, Toyama, 59-64, doi:10.1109/ISMVL.2013.38, 2013.

Li, B., Li, W., Liu, H., Yao, R., Tan, M., Jing, S., and Ma, X.: Physiological Expression of Human Thermal Comfort to Indoor Operative Temperature in the Non-HVAC Environment, Indoor Built Environ., 19, 221-229, doi:10.1177/1420326X10365213, 2010.

Nicol, F. and Humphreys, M.: Derivation of the adaptive equations for thermal comfort in free-running buildings in European standard EN15251, Build. Environ., 45, 11-17, doi:10.1016/j.buildenv.2008.12.013, 2010.

openHAB - Supported Technologies, availabe at: http://www. openhab.org/features/supported-technologies.html, last access: 5 January 2017.

Renn, O.: Akzeptanzforschung: Technik in der gesellschaftlichen Auseinandersetzung, Chemie in unserer Zeit, 20, 44-52, 1986.

Shah, N. and Hewitt, N.: High temperature heat pump operational experience as a retrofit technology in domestic sector. IEEE International Conference on Engineering, Technology and Innovation/International Technology Management Conference, Belfast, 7 pp., doi:10.1109/ICE.2015.7438691, 22-24 June 2015.

Simone, A., Babiak, J., Bullo, M., Landkilde, G., and Olesen, B. W.: Operative temperature control of radiant surface heating and cooling systems, Proc. 9th REHVA World Congress Clima 2007 "WellBeing Indoors", Helsinki, 8 pp., 10-14 June 2007.

Smirek, L., Zimmermann, G., and Beigl, M.: Just a Smart Home or Your Smart Home - A Framework for Personalized User Interfaces Based on Eclipse Smart Home and Universal Remote Console, Proc. Comput. Sci., 98, 107-116, doi:10.1016/j.procs.2016.09.018, 2016. 